\title{
Synthesis and Evaluation of Anticancer Activity of O-allylchalcone Derivatives
}

\section{Bathélémy Ngameni ${ }^{1 *}$, Victor Kuete ${ }^{2}$, Pantaleon Ambassa ${ }^{3}$, kamga Justin ${ }^{3}$, Moungang Luciane Marlyse $^{4}$, Abdou Tchoukoua $^{3}$, René Roy $^{5}$, Bonaventure Tchaleu Ngadjui ${ }^{1,3}$ and Murayama Tetsuya ${ }^{6}$}

${ }^{1}$ Department of Pharmaceutical Sciences and Traditional Pharmacopoeia, Faculty of Medicine and Biomedical Sciences, University of Yaoundé I, Cameroon ${ }^{2}$ Department of Biochemistry, Faculty of Science, University of Dschang, Cameroon

${ }^{3}$ Department of Organic Chemistry, Faculty of Science, University of Yaoundé I, Cameroon

${ }^{4}$ Department of Biology and Animal Physiology, Faculty of Science, University of Yaoundé I, Cameroon

${ }^{5}$ Department of Chemistry, Université du Québec à Montréal, Québec, Canada

${ }^{6}$ Department of Chemistry, Faculty of Agriculture, University of Yamagata, Japan

\begin{abstract}
A large number of novel O-allylchalcones were synthesized by Claisen Schmidt condensation reaction of $\mathrm{O}$-allylvanillin 3 with appropriate substituted acetophenones $4 \mathrm{a}-\mathrm{h}$. These model chalcones $5 \mathrm{a}-\mathrm{h}$ and their precursor O-allylvanillin were screened for their in vitro cytotoxic activity against four human cancer cell lines. The most potent compound in this series with the $\mathrm{IC}_{50}$ values below or around $10 \mu \mathrm{M}$ were $5 \mathrm{f}$ against THP-1 cells $(10.42 \mu \mathrm{M})$ and $5 \mathrm{~g}$ against THP-1 $(4.76 \mu \mathrm{M})$, DU-145 $(5.21 \mu \mathrm{M})$, HL60 $(7.90 \mu \mathrm{M})$, Hep-G2 $(10.12 \mu \mathrm{M})$ and MCF-7 (10.32 $\mu \mathrm{M})$.
\end{abstract}

Keywords: Synthesis; O-allylchalcones; Anticancer; Structureactivity relationship

\section{Introduction}

There is a currently a good deal of interest in the health benefits of phytochemicals, in particular prenylated and allylated flavonoids. Chalcones (1,3-diaryl-2-propen-1-ones) and their derivatives are important intermediates of flavonoid synthetic pathway. Chalcones, one of the major classes of natural products with widespread distribution in fruits, vegetables, spices, tea and soy based foodstuff have also been the subject of great interest for their interesting pharmacological activities [1]. Chemically they can be considered open-chain flavonoids in which the two aromatic rings are joined by a three-carbon $\alpha, \beta$-unsaturated carbonyl system. Chalcones have also been reported to possess many useful biological and pharmacological properties, including antibacterial $[2,3]$, antimalarial $[4,5]$, antifungal [6], antiviral $[7,8]$, anti-inflammatory $[9,10]$, and anticancer $[11,12]$ properties. A good safety profile, possibility of oral administration [13] and easy synthesis are the major factors contributing to the increasing interest in exploring the pharmacological activities of chalcones. Chalcones comprise one of the main classes of natural small molecules with very promising anticancer activity, related to their ability to inhibit tubulin polymerization [14]. Most of the anticancer agents, of natural or synthetic origin exhibit enone function in their structure $[15,16]$. Also, synthesized chalcones holding allylic substitutions were recently reported as potent antimicrobial and antioxidant agents $[17,18]$. In addition, the substitution of ring B with electron withdrawing groups like methoxy or hydroxy group improve the antiproliferative activity against human colon HT-29 cancer cell line [19].

Prompted by all these observations, we report herein the synthesis of novel $O$-allylchalcones, bearing various substituents with potent activity against Human Hep-G2 hepatocarcinoma, breast carcinoma MCF-7, prostate carcinoma DU-145, and acute monocytic leukemia THP-1 and HL-60 cell lines. The structure-activity relationships are also discussed.

\section{Materials and Methods}

\section{Chemistry}

IR spectra were determined with a Perkin Elmer FT-IR spectrophotometer. ${ }^{1} \mathrm{H}$ and ${ }^{13} \mathrm{C}$ NMR spectra were recorded with Bruker WM-300 in the $\mathrm{CDCl}_{3}$ at 300 and $75 \mathrm{MHz}$, respectively using
TMS as the internal standard. All chemical shifts are reported on $\delta$ scale. Mass spectra were obtained using a Varian MAT-311A. Thinlayer chromatography (TLC) was carried out using Merck silica gel 60 F-254 plates (layer thickness $0.25 \mathrm{~mm}$ ) and all solvents were distilled prior to use.

\section{Synthesis}

Compounds $5 \mathrm{a}-\mathrm{h}$ were synthesized by the condensation reaction of compound 3 with different substituted acetophenones $4 \mathrm{a}-\mathrm{h}$. The main intermediate 3 was prepared from vanillin 1 and allylbromide 2 in the presence of potassium carbonate in anhydrous acetone.

\section{Biology}

Cytotoxicity assay: Cell lines and treatment: The effect of synthesized compounds on cell growth was determined on five human tumor cells including Hep-G2 hepatocarcinoma, breast carcinoma MCF-7, prostate carcinoma DU-145, and acute monocytic leukemia THP-1 and HL-60 cell lines, obtained from National Cancer Institute, USA. THP-1 and HL-60 were maintained in RPMI medium while Hep-G2, MCF-7 and DU-145 were cultured in MEM medium. All media used were supplemented with $10 \%$ fetal bovine serum (FBS), 100 $\mathrm{IU} / \mathrm{mL}$ penicillin. The cell lines were maintained under standard cell culture conditions at $37^{\circ} \mathrm{C}$ and $5 \% \mathrm{CO}_{2}$ in a humidified environment.

The cytotoxicity of the samples against the five studied human cell lines was determined using Sulphorhodamine B (SRB) assay as previously described [20]. The cells were incubated at $37{ }^{\circ} \mathrm{C}$ in an atmosphere of $5 \% \mathrm{CO}_{2}$ and $95 \%$ relative humidity in a $\mathrm{CO}_{2}$ incubator. Doxorubicin was used as positive reference. Suitable controls with

*Corresponding author: Bathelemy Ngameni, Department of Pharmaceutical Sciences and Traditional Pharmacopoeia, Faculty of Medicine and Biomedical Sciences, University of Yaoundé I, Cameroon, P.O. Box 8664, Tel: +237 76480440; Fax: +237 22221873; E-mail: bath_ngameni@yahoo.fr

Received June 17, 2013; Accepted July 26, 2013; Published July 28, 2013

Citation: Ngameni B, Kuete V, Ambassa P, Justin K, Marlyse ML, et al. (2013) Synthesis and Evaluation of Anticancer Activity of O-allylchalcone Derivatives. Med chem 3: 233-237. doi:10.4172/2161-0444.1000144

Copyright: (c) 2013 Ngameni B, et al. This is an open-access article distributed under the terms of the Creative Commons Attribution License, which permits unrestricted use, distribution, and reproduction in any medium, provided the original author and source are credited. 
equivalent concentration of DMSO were also included. The optical density (OD) was recorded using a 96 well plate reader, and growth inhibition was calculated [20]. A preliminary study was first carried out with compounds (Table $1,100 \mu \mathrm{M}$ ) and doxorubicin (at $50 \mu \mathrm{M})$ to detect if samples were able to inhibit the proliferation of more that $50 \%$ of the cells. Then samples were serially diluted and tested against other cell lines for $\mathrm{IC}_{50}$ determination. $\mathrm{IC}_{50}$ is the concentration of sample required to inhibit $50 \%$ of the cell proliferation after $72 \mathrm{~h}$ incubation and was calculated by plotting the percentage survival versus the concentration, using Microsoft Excel. For all samples, each compound concentration was tested thrice in triplicates.

\section{Experimental}

\section{4-Allyloxy-3-methoxybenzaldehyde or $O$-allylvanillin (3)}

To $0.304 \mathrm{~g}(1.99 \mathrm{mmol})$ of vanillin in acetone $(8 \mathrm{~mL})$ was added $\mathrm{K}_{2} \mathrm{CO}_{3}(0.1203 \mathrm{~g})$ followed by allylbromide $(0.12 \mathrm{~mL}, \mathrm{~d}=1.43,0.1772$ $\mathrm{g}, 1.46 \mathrm{mmol}$ ). The reaction mixture was heated to reflux for 4 hours or left at room temperature for 48 hours. At the end of the reaction, the solvent was evaporated under reduced pressure and the residue is diluted in water $(40 \mathrm{ml} \mathrm{x} 3)$. The aqueous mixture was extracted with Ethyl Acetate $(\mathrm{EA})(3 \times 60 \mathrm{~mL})$ and the extract was dried by anhydrous $\mathrm{Na}_{2} \mathrm{SO}_{4}$. After evaporation of the solvent and purification by column chromatography on silica gel eluting with Hexane-Ethyl Acetate (HexEA) system of increasing polarity, product 3 was obtained $(725 \mathrm{mg}$, yield $70 \%$ in Hex-EA 87.5:12.5). ${ }^{1} \mathrm{H}$ NMR $\left(600 \mathrm{MHz}, \mathrm{CDCl} 3, \mathrm{Me}_{4} \mathrm{Si}\right)$ $\delta 3.79(3 \mathrm{H} ; \mathrm{s}), 4.45(2 \mathrm{H} ; \mathrm{t} ; J=5.7 \mathrm{~Hz}), 5.23(1 \mathrm{H} ; \mathrm{dd} ; J=1.5$ and $14,4 \mathrm{~Hz})$, $5.34(1 \mathrm{H}$; dd; $J=1.5$ and $14.4 \mathrm{~Hz}), 5.95(1 \mathrm{H}$; dd; $J=5.4$ and $1.5 \mathrm{~Hz}), 6.84$ $(1 \mathrm{H} ; \mathrm{d} ; J=8.4 \mathrm{~Hz}), 7.28(1 \mathrm{H} ; \mathrm{dd} ; J=7.8$ and $1.5 \mathrm{~Hz}), 7.30(1 \mathrm{H} ; \mathrm{d} ; J=1.5$ $\mathrm{Hz}), 9.70(1 \mathrm{H} ; \mathrm{s}) ;$ ESIMS m/z $193.2[\mathrm{M}+\mathrm{H}]^{+}$. HREIMS (m/z): 192.0776 $\left[\mathrm{M}^{+}\right]$(calcd for $\left.\mathrm{C}_{11} \mathrm{H}_{12} \mathrm{O}_{3}, 192.0786\right)$.

\section{4-allyloxy-3-methoxychalcone (5a)}

To a solution of acetophenone $(0.18 \mathrm{~mL}, 3.41 \mathrm{mmol}, \mathrm{d}=1.0266)$ in methanol $(30 \mathrm{~mL})$ was added first $O$-allylvanillin $(110 \mathrm{mg}, 0.57$ $\mathrm{mmol})$ and then an aqueous solution of $\mathrm{KOH}(50 \%, 1 \mathrm{~mL} / \mathrm{mmol}$ of acetophenone) or $3.41 \mathrm{~mL}$. The reaction mixture was refluxed at $70^{\circ} \mathrm{C}$ for 5 hours or left at room temperature for 15 hours. At the end of the reaction the mixture was diluted with water $(30 \mathrm{~mL})$ and extracted with $\mathrm{CH}_{2} \mathrm{Cl}_{2}(3 \times 70 \mathrm{~mL})$ and then the extract was washed with water $(50 \mathrm{~mL})$ and saturated with $\mathrm{NaCl}$ solution. The organic phase was dried with $\mathrm{Na}_{2} \mathrm{SO}_{4}$ and the solvent evaporated under reduced pressure. After purification of the residue by Column Chromatography and Thin Layer Chromatography preparative on silica gel (Hex-EA 9:1), compound 5a was obtained pure (103.2 mg, yield $28 \%$ in Hex-EA 80:20). IR $\left(\mathrm{CHCl}_{3}\right)$ : $v_{\max } \mathrm{cm}^{-1}: 1654,1579,1257 .{ }^{1} \mathrm{H}$ NMR $\left(300 \mathrm{MHz}, \mathrm{CDCl}_{3}, \mathrm{Me}_{4} \mathrm{Si}\right): \delta 3.70$ $(3 \mathrm{H} ; \mathrm{s}), 4.33(2 \mathrm{H} ; \mathrm{d} ; J=6.5 \mathrm{~Hz}), 5.47(1 \mathrm{H} ; \mathrm{dd} ; J=17.5$ and $10.9 \mathrm{~Hz}), 5.55$ $(1 \mathrm{H}$; dd; $J=17.5$ and $0.9 \mathrm{~Hz}), 6.18(1 \mathrm{H} ; \mathrm{m}), 6.99(1 \mathrm{H} ; \mathrm{d} ; J=8.4 \mathrm{~Hz})$, $7.20(1 \mathrm{H}$; dd ; J=8.4 and $1.5 \mathrm{~Hz}), 7.27(1 \mathrm{H} ; \mathrm{d} ; \mathrm{J}=1.5 \mathrm{~Hz}), 7.48(1 \mathrm{H}$; d; $\mathrm{J}=15.9 \mathrm{~Hz}), 7.50(1 \mathrm{H} ; \mathrm{m} ; \mathrm{J}=8.1$ and $2.1 \mathrm{~Hz}), 7.52(1 \mathrm{H} ; \mathrm{dd} ; \mathrm{J}=8.1$ and $1.5 \mathrm{~Hz}), 7.7\left(1 \mathrm{H}\right.$; d; J=15.6 Hz), $7.81(1 \mathrm{H}$; dd; J=8.4 and $1.5 \mathrm{~Hz}) ;{ }^{13} \mathrm{C}$ NMR $\left(75 \mathrm{MHz}, \mathrm{CDCl}_{3}, \mathrm{Me}_{4} \mathrm{Si}\right) \delta 59.9 ; 69.5 ; 110.4 ; 112.8 ; 118.4 ; 120.0$; $122.9 ; 127.4 ; 128.3 ; 129.7 ; 132.5 ; 132.6 ; 138.4 ; 144.9 ; 149.5 ; 150.5 ; 190.5$; ESIMS m/z 295.3 [M + H] $]^{+}$. HREIMS (m/z): $294.1260\left[\mathrm{M}^{+}\right](\mathrm{calcd}$ for $\left.\mathrm{C}_{19} \mathrm{H}_{18} \mathrm{O}_{3}, 294.1256\right)$.

\section{4-allyloxy-2',3-diméthoxychalcone (5b)}

To a solution of 2-methoxyacetophenone $(27,84 \mu \mathrm{l}, \mathrm{d}=1.090)$ in ethanol $(5 \mathrm{~mL})$ was added first $O$-allylvanillin $(38.8 \mathrm{mg}, 0.20$ $\mathrm{mmol})$ and secondly an aqueous solution $\mathrm{KOH}(50 \%, 1 \mathrm{~mL} / \mathrm{mmol}$ of acetophenone) or $0.20208 \mathrm{~mL}$. The mixture was refluxed at $70^{\circ} \mathrm{C}$ for 5 hours or left at room temperature for 15 hours. We obtained the product 5b (42.4 mg, yield 65\% in Hex-EA 87.5:12.5) after separation of the residue of the reaction. IR $\left(\mathrm{CHCl}_{3}\right): v_{\max } \mathrm{cm}^{-1}: 2368.9,2333.6$, 1653.5, 1594.8, 1250.8, 1019.5; ${ }^{1} \mathrm{H}$ NMR $\left(300 \mathrm{MHz}, \mathrm{CDCl}_{3}, \mathrm{Me}_{4} \mathrm{Si}\right): \delta$ $3.93(3 \mathrm{H} ; \mathrm{s}), 3.95(3 \mathrm{H} ; \mathrm{s}), 4.70(2 \mathrm{H} ; \mathrm{d} ; \mathrm{J}=1.2 \mathrm{~Hz}), 5.36(1 \mathrm{H} ; \mathrm{dd} ; \mathrm{J}=13.5$ and $12.6 \mathrm{~Hz}), 5.45(1 \mathrm{H}$; dd; $\mathrm{J}=13.5 \mathrm{and} 1.8 \mathrm{~Hz}), 6.10(1 \mathrm{H} ; \mathrm{m}), 6.91(1 \mathrm{H}$ d; J=8,4 Hz), $7.04(1 \mathrm{H}$; d; J=15.9 Hz), $7.10(1 \mathrm{H}$; d; J=8.7 and $1.5 \mathrm{~Hz})$, $7.15(1 \mathrm{H} ; \mathrm{d} ; \mathrm{J}=1.8 \mathrm{~Hz}), 7.16(1 \mathrm{H}$; dd; $\mathrm{J}=8.7$ and $1.8 \mathrm{~Hz}), 7.23(1 \mathrm{H} ; \mathrm{m})$ , $7.49(1 \mathrm{H}$; dd; $J=9.0$ and $1.5 \mathrm{~Hz}), 7.58(1 \mathrm{H} ; \mathrm{d} ; \mathrm{J}=16.2 \mathrm{~Hz}), 7.62(1 \mathrm{H}$; d; J=7.8 and $1.5 \mathrm{~Hz}) ;{ }^{13} \mathrm{C}$ NMR $\left(75 \mathrm{MHz}, \mathrm{CDCl}_{3}, \mathrm{Me}_{4} \mathrm{Si}\right): \delta$ 55.3; 55.5; $69.3 ; 110.1 ; 111.1 ; 112.4 ; 117.9 ; 120.2 ; 122.2 ; 124.8 ; 127.8 ; 129.1 ; 129.6$; $132.0 ; 132.3 ; 143.4 ; 149.0 ; 149.7 ; 157.4 ; 192,8 ;$ ESIMS m/z $325[\mathrm{M}+\mathrm{H}]^{+}$ HREIMS (m/z): $324.1365\left[\mathrm{M}^{+}\right]$(calcd for $\mathrm{C}_{20} \mathrm{H}_{20} \mathrm{O}_{4}, 324.1362$ ).

\section{4-allyloxy-3-methoxy-2', 4'-diméthylchalcone (5c)}

To a solution of 2,4-dimethoxyacetophenone $(48.46 \mu \mathrm{l}, 0.33 \mathrm{mmol}$, $\mathrm{d}=0.997)$ in ethanol $(7 \mathrm{~mL})$ were added first $O$-allylvanillin $(62.6 \mathrm{mg}$, $0.33 \mathrm{mmol})$, and secondly an aqueous solution of $\mathrm{KOH}(50 \%, 1 \mathrm{~mL} /$ $\mathrm{mmol}$ ) or $0.32604 \mathrm{~mL}$. The mixture was left at room temperature for 23 hours. After separation and purification of the residue of the reaction, the product $5 \mathrm{c}$ was obtained (53.5 mg, yield $82 \%$ in Hex-EA 85:15). IR $\left(\mathrm{CHCl}_{3}\right): v_{\max } \mathrm{cm}^{-1}: 2917.5,2369.6,1590.9,1508.7,1260.7,1139.2$ 1014.7; ${ }^{1} \mathrm{H}$ NMR $\left(300 \mathrm{MHz}, \mathrm{CDCl}_{3}, \mathrm{Me}_{4} \mathrm{Si}\right.$ ): $\delta 2.55$ (3H; s), $2.60(3 \mathrm{H} ; \mathrm{s})$, $4.10(3 \mathrm{H} ; \mathrm{s}), 4.81(2 \mathrm{H} ; \mathrm{d} ; \mathrm{J}=1.5 \mathrm{~Hz}), 5.48(1 \mathrm{H} ; \mathrm{dd} ; \mathrm{J}=9.0$ and $1.5 \mathrm{~Hz}), 5.61$ $(1 \mathrm{H}$; dd; J=15.9 and $1.5 \mathrm{~Hz}), 6.24(1 \mathrm{H} ; \mathrm{m}), 7.05(1 \mathrm{H} ; \mathrm{d} ; \mathrm{J}=9.0 \mathrm{~Hz}), 7.20$ $(1 \mathrm{H} ; \mathrm{d} ; \mathrm{J}=15.9 \mathrm{~Hz}), 7.26(2 \mathrm{H} ; \mathrm{d} ; \mathrm{J}=1.8 \mathrm{~Hz}), 7.28(1 \mathrm{H} ; \mathrm{dd} ; \mathrm{J}=8.4$ and 1.8 $\mathrm{Hz}), 7.29(1 \mathrm{H}$; dd; J=8.1 and $1.8 \mathrm{~Hz}), 7.58(1 \mathrm{H} ; \mathrm{d} ; \mathrm{J}=8.1 \mathrm{~Hz}), 7.59(1 \mathrm{H}$; d; J=15.9 Hz); ${ }^{13} \mathrm{C}$ NMR $\left(75 \mathrm{MHz}, \mathrm{CDCl} 3, \mathrm{Me}_{4} \mathrm{Si}\right) \delta 19.8 ; 20.9 ; 55.4 ;$ $69.2 ; 109.8 ; 112.3 ; 117.9 ; 122.3 ; 124.4 ; 125.5 ; 127.4 ; 127.9 ; 131.6 ; 132.2$ $135.9 ; 136.7 ; 140.11 ; 145.0 ; 149.0 ; 149.8 ; 195.7 ;$ ESIMS m/z $323[\mathrm{M}+$ $\mathrm{H}]^{+}$. HREIMS (m/z): $322.1566\left[\mathrm{M}^{+}\right]\left(\right.$calcd for $\left.\mathrm{C}_{21} \mathrm{H}_{22} \mathrm{O}_{3}, 322.1569\right)$.

\section{4-allyloxy-3',3-diméthoxychalcone (5d)}

To a solution of 3-methoxyacetophenone $(71.5 \mu \mathrm{l}, 0.52 \mathrm{mmol}$, $\mathrm{d}=1.094)$ in ethanol $(7 \mathrm{ml})$ was added first $O$-allylvanillin $(100 \mathrm{mg}, 0.52$ $\mathrm{mmol}$ ) and secondly a $\mathrm{KOH}$ solution $(50 \%, 1 \mathrm{~mL} / \mathrm{mmol})$ or $0.52 \mathrm{~mL}$. The mixture is stirred in a nitrogen atmosphere at room temperature for 21 hours. After separation and purification of the residue of the reaction by $\mathrm{CC}$, the product $5 \mathrm{~d}$ was obtained $(64.6 \mathrm{mg}$, yield $38 \%$ in Hex-EA 85:15). IR spectrum $\left(\mathrm{CHCl}_{3}\right): v_{\text {max }} \mathrm{cm}^{-1}: 2917.2,2361.7,1658.3$, $1576.3,1508.7,1260.5,1140.2,1029.9 ;{ }^{1} \mathrm{H}$ NMR $\left(300 \mathrm{MHz}, \mathrm{CDCl}_{3}\right.$ $\left.\mathrm{Me}_{4} \mathrm{Si}\right): \delta 3.75(3 \mathrm{H} ; \mathrm{s}), 3.81(3 \mathrm{H} ; \mathrm{s}), 4.53(2 \mathrm{H} ; \mathrm{d} ; \mathrm{J}=5.1 \mathrm{~Hz}), 5.20(1 \mathrm{H} ; \mathrm{dd}$ $\mathrm{J}=15.9$ and $1.2 \mathrm{~Hz}), 5.31(1 \mathrm{H} ; \mathrm{dd} ; \mathrm{J}=15.9$ and $9.6 \mathrm{~Hz}), 5.92(1 \mathrm{H} ; \mathrm{m}), 6.75$ $(1 \mathrm{H} ; \mathrm{d} ; \mathrm{J}=8.4 \mathrm{~Hz}), 6.98(1 \mathrm{H} ; \mathrm{d} ; \mathrm{J}=1.5 \mathrm{~Hz}), 7.06(1 \mathrm{H} ; \mathrm{d} ; \mathrm{J}=1.8 \mathrm{~Hz}), 7.07$ $(1 \mathrm{H}$; dd; $\mathrm{J}=8.4$ and $1.8 \mathrm{~Hz}), 7.21(1 \mathrm{H}$; ddd; $\mathrm{J}=9.3,1.6$ and $1.5 \mathrm{~Hz}), 7.27$ $(1 \mathrm{H} ; \mathrm{d} ; \mathrm{J}=15.9 \mathrm{~Hz}), 7.29(1 \mathrm{H}$; dd; $\mathrm{J}=9.3$ and $8.1 \mathrm{~Hz}), 7.46(1 \mathrm{H} ; \mathrm{dd} ; \mathrm{J}=8.1$ and $1.5 \mathrm{~Hz}), 7.63(1 \mathrm{H} ; \mathrm{d} ; \mathrm{J}=15.6 \mathrm{~Hz}) ;{ }^{13} \mathrm{C} \mathrm{NMR}\left(75 \mathrm{MHz}, \mathrm{CDCl}_{3}, \mathrm{Me}_{4} \mathrm{Si}\right)$ : $\delta 55.8 ; 56.4 ; 70.1 ; 110.8 ; 113.2 ; 113.3 ; 118.8 ; 119.31 ; 120.4 ; 121.3 ; 123.4$; $128.4 ; 129.9 ; 133.04 ; 140.2 ; 145.4 ; 149.9 ; 150.8 ; 160.2 ; 190.6 ;$ ESIMS m/z $649.3[2 \mathrm{M}+\mathrm{H}]^{+}, 325.2[\mathrm{M}+\mathrm{H}]^{+}$. HREIMS $(\mathrm{m} / \mathrm{z}): 324.1358\left[\mathrm{M}^{+}\right](\mathrm{calcd}$ for $\mathrm{C}_{20} \mathrm{H}_{20} \mathrm{O}_{4}, 324.1362$ ).

\section{4-allyloxy-3-methoxy-3', 4'-diméthylchalcone (5e)}

To a solution of 3,4-dimethylacetophenone $(77.11 \mu \mathrm{l}, 0.52 \mathrm{mmol}$, $\left.\mathrm{d}=1.001 ; \mathrm{n}_{\mathrm{D}}{ }^{20}: 1.538\right)$ in ethanol $(7 \mathrm{~mL})$ was first added $O$-allylvanillin (100 mg, $0.52 \mathrm{mmol}$ ), and secondly a $0.52 \mathrm{~mL}$ solution of $\mathrm{KOH}$ $(50 \%, 1 \mathrm{~mL} / \mathrm{mmol})$ that is $0.52 \mathrm{~mL}$. The mixture was stirred at room temperature for 26 hours. After separation and purification of the residue of the reaction, we obtained the compound $5 \mathrm{e}(68.8 \mathrm{mg}$, yield $41 \%$ in Hex-EA 85:15). IR spectrum $\left(\mathrm{CHCl}_{3}\right): v_{\max } \mathrm{cm}^{-1}: 2919.0,2361.3$, 1653.5, 1508.7, 1259.7, 1136.7; ${ }^{1} \mathrm{H}$ NMR (300 MHz, $\left.\mathrm{CDCl}_{3}, \mathrm{Me}_{4} \mathrm{Si}\right) \delta 2.2$ $(6 \mathrm{H} ; \mathrm{s}), 3.8(3 \mathrm{H} ; \mathrm{s}), 4.50(2 \mathrm{H} ; \mathrm{d} ; \mathrm{J}=5.6 \mathrm{~Hz}), 5.20(1 \mathrm{H} ; \mathrm{dd} ; \mathrm{J}=17.3$ and 9.6 $\mathrm{Hz}), 5.23(1 \mathrm{H} ; \mathrm{dd} ; \mathrm{J}=17.3$ and $0.5 \mathrm{~Hz}), 5.9(1 \mathrm{H} ; \mathrm{m}), 6.74(1 \mathrm{H} ; \mathrm{d} ; \mathrm{J}=8.6$ 
$\mathrm{Hz}), 7.02(1 \mathrm{H} ; \mathrm{dd} ; \mathrm{J}=8.6$ and $1.7 \mathrm{~Hz}), 7.05(1 \mathrm{H} ; \mathrm{d} ; \mathrm{J}=1.7 \mathrm{~Hz}), 7.10(1 \mathrm{H} ; \mathrm{d}$ ; J=8.7 Hz), $7.24(1 \mathrm{H} ; \mathrm{d} ; \mathrm{J}=15.3 \mathrm{~Hz}), 7.59(1 \mathrm{H} ; \mathrm{d} ; \mathrm{J}=1.8 \mathrm{~Hz}), 7.60(1 \mathrm{H} ; \mathrm{d}$; $\mathrm{J}=15.6 \mathrm{~Hz}), 7.64(1 \mathrm{H} ; \mathrm{dd} ; \mathrm{J}=8.7$ and $1.8 \mathrm{~Hz}),{ }^{13} \mathrm{C} \mathrm{NMR}(75 \mathrm{MHz}, \mathrm{CDCl}$ $\left.{ }_{3}, \mathrm{Me}_{4} \mathrm{Si}\right) \delta 20.0 ; 20.3 ; 56.2 ; 69.9 ; 110.9 ; 113.3 ; 118.6 ; 120.5 ; 122.9 ; 126.4$; $128.4 ; 129.8 ; 129.9 ; 132.9 ; 136.5 ; 137.2 ; 142.4 ; 144.6 ; 149.7 ; 150.5 ; 190.5$; ESIMS m/z 323,3 $[\mathrm{M}+\mathrm{H}]^{+}$. HREIMS (m/z): $322.1562\left[\mathrm{M}^{+}\right]$(calcd for $\left.\mathrm{C}_{21} \mathrm{H}_{22} \mathrm{O}_{3}, 322.1569\right)$.

\section{4-allyloxy-3-methoxy-3'-methylchalcone (5f)}

To a solution of 3-methylacetophenone $(70.88 \mu \mathrm{l}, 0.52 \mathrm{mmol}, \mathrm{d}$ $\left.=0.986 ; \mathrm{n}_{\mathrm{D}}{ }^{20}=1.529\right)$ in ethanol $(7 \mathrm{~mL})$ was first added $O$-allylvanillin (100 mg, $0.52 \mathrm{mmol})$, and secondly a $0.52 \mathrm{~mL}$ solution of $\mathrm{KOH}(50 \%, 1$ $\mathrm{mL} / \mathrm{mol}$ ) that is $0.52 \mathrm{~mL}$. The mixture was stirred at room temperature for 24 hours. After separation and purification of the residue of the reaction product was obtained $\mathbf{5 f}(96.3 \mathrm{mg}$, yield $60 \%$ in Hex-EA 88:12). IR spectrum $\left(\mathrm{CHCl}_{3}\right): v_{\text {max }} \mathrm{cm}^{-1}: 2927.1,2367.7,1655.3,1578.9$, $1506.4,1256.9,1132.1 ;{ }^{1} \mathrm{H}$ NMR $\left(300 \mathrm{MHz}, \mathrm{CDCl}_{3}, \mathrm{Me}_{4} \mathrm{Si}\right) \delta 2,31(3 \mathrm{H}$; s), $3.82(3 \mathrm{H} ; \mathrm{s}), 4.54(2 \mathrm{H} ; \mathrm{d} ; \mathrm{J}=1.4 \mathrm{~Hz}), 5.20(1 \mathrm{H} ; \mathrm{dd} ; \mathrm{J}=13.0$ and $6.6 \mathrm{~Hz})$ , $5.30(1 \mathrm{H} ; \mathrm{dd} ; \mathrm{J}=13.0$ and $1.5 \mathrm{~Hz}), 5.96(1 \mathrm{H} ; \mathrm{m}), 6.77(1 \mathrm{H} ; \mathrm{d} ; \mathrm{J}=8.7 \mathrm{~Hz})$, $7.04(1 \mathrm{H} ; \mathrm{d} ; \mathrm{J}=1.8 \mathrm{~Hz}), 7.06(1 \mathrm{H}$; dd; J=8.7 and $1.8 \mathrm{~Hz}), 7.10(1 \mathrm{H}$; d; $\mathrm{J}=15.9 \mathrm{~Hz}), 7.23(1 \mathrm{H}$; dd; J=8.4 and $8.1 \mathrm{~Hz}), 7.27(1 \mathrm{H}$; ddd; J=8.1; 1.8 and $1.5 \mathrm{~Hz}), 7.6(1 \mathrm{H}$; ddd; $\mathrm{J}=8.4 ; 1.8$ and $1.5 \mathrm{~Hz}), 7.62(1 \mathrm{H} ; \mathrm{d} ; \mathrm{J}=15.9$ $\mathrm{Hz}), 7.65(1 \mathrm{H}$; dd; $\mathrm{J}=1.8$ and $1.5 \mathrm{~Hz}),{ }^{13} \mathrm{C} \mathrm{NMR}\left(75 \mathrm{MHz}, \mathrm{CDCl}_{3}, \mathrm{Me}_{4} \mathrm{Si}\right)$ $\delta 21.0 ; 55.6 ; 69.3 ; 110.1 ; 112.5 ; 118.0 ; 119.9 ; 122.4 ; 125.2 ; 127.6 ; 128.0$; $128.5 ; 131.1 ; 132.9 ; 137.9 ; 138.1 ; 144.4 ; 149.1 ; 149.9 ; 190.4 ;$ ESIMS m/z 309,3 $[\mathrm{M}+\mathrm{H}]^{+}$. HREIMS (m/z): $308.1407\left[\mathrm{M}^{+}\right]\left(\right.$calcd for $\mathrm{C}_{20} \mathrm{H}_{20} \mathrm{O}_{3}$, 308.1412).

\section{4-allyloxy-3-methoxy-2'-methoxychalcone (5g)}

To a solution of 2-methylacetophenone $(139.769 \mu \mathrm{l}, 1.04 \mathrm{mmol}, \mathrm{d}=$ $\left.1.026, \mathrm{n}_{\mathrm{D}}{ }^{20}=1.5318\right)$ in ethanol $(7 \mathrm{~mL})$ was first added to $O$-allylvanillin (60 mg, $0.31 \mathrm{mmol}$ ), and secondly a $1.04166 \mathrm{~mL}$ solution of $\mathrm{KOH} \mathrm{(50 \% ,}$ $1 \mathrm{~mL} / \mathrm{mol}$ ). The reaction mixture was stirred room temperature for 24 hours. After separation and purification of the residue of the reaction, the product $5 \mathrm{~g}$ was obtained ( $41.9 \mathrm{mg}$, yield $44 \%$ in Hex-EA 92:8). IR spectrum $\left(\mathrm{CHCl}_{3}\right): v_{\max } \mathrm{cm}^{-1}: 2365.7,2336.6,1633.9,1590.9,1508.7$, $1262.9,1143.8 ;{ }^{1} \mathrm{H}$ NMR (300 MHz, $\left.\mathrm{CDCl}_{3}, \mathrm{Me}_{4} \mathrm{Si}\right) \delta 2.35$ (3H; s), 3.83 $(3 \mathrm{H} ; \mathrm{s}), 4.57(2 \mathrm{H}$; d; J=1.6 Hz), $6.79(1 \mathrm{H} ; \mathrm{d}$; J=1.8 Hz) , $5.23(1 \mathrm{H}$; dd; $\mathrm{J}=14.1$ and $5.7 \mathrm{~Hz}), 5.34(1 \mathrm{H} ; \mathrm{dd} ; \mathrm{J}=14.1$ and $1.8 \mathrm{~Hz}), 5.90(1 \mathrm{H} ; \mathrm{m}), 6.81$ $(1 \mathrm{H} ; \mathrm{d} ; \mathrm{J}=1.8 \mathrm{~Hz}), 6.92(1 \mathrm{H} ; \mathrm{d} ; \mathrm{J}=16.2 \mathrm{~Hz}), 7.18(1 \mathrm{H} ; \mathrm{dd} ; \mathrm{J}=9.0$ and 1.5 $\mathrm{Hz}), 7,20(1 \mathrm{H}$; dd; J=8.4 and $1.8 \mathrm{~Hz}), 7.21(1 \mathrm{H} ; \mathrm{m} ; \mathrm{J}=9.0$ and $2.1 \mathrm{~Hz})$, $7.29(1 \mathrm{H} ; \mathrm{m} ; \mathrm{J}=9.0$ and $2.1 \mathrm{~Hz}), 7.30(1 \mathrm{H}$; d; J=15.9 Hz), $7.40(1 \mathrm{H} ; \mathrm{d}$; $\mathrm{J}=9.0$ and $2.1 \mathrm{~Hz}),{ }^{13} \mathrm{C} \mathrm{NMR}\left(75 \mathrm{MHz}, \mathrm{CDCl}_{3}, \mathrm{Me}_{4} \mathrm{Si}\right) \delta 20.02 ; 55.9 ; 69.7$; $110.2 ; 112.8 ; 118.4 ; 122.9 ; 124.9 ; 125.4 ; 127.6 ; 127.8 ; 130.1 ; 131.1 ; 132.6$; $136.6 ; 139.3 ; 146.2 ; 149.3 ; 150.4 ; 196.8 ;$ ESIMS m/z $309.3[\mathrm{M}+\mathrm{H}]^{+}$. HREIMS (m/z): $308.1403\left[\mathrm{M}^{+}\right]$(calcd for $\left.\mathrm{C}_{20} \mathrm{H}_{20} \mathrm{O}_{3}, 308.1412\right)$.

\section{4-allyloxy-3, 4'-diméthoxychalcone $(5 \mathrm{~h})$}

To a solution of 4-methylacetophenone $(151.8 \mu \mathrm{l}, 1.02 \mathrm{mmol})$ in ethanol $(5 \mathrm{~mL})$ was first added to $O$-allylvanillin $(75 \mathrm{mg}, 0.39 \mathrm{mmol})$, and secondly a $1.0107 \mathrm{~mL}$ solution of $\mathrm{KOH}(50 \%, 1 \mathrm{~mL} / \mathrm{mol})$. The reaction mixture was stirred at room temperature for 24 hours. After separation and purification of the residue of the reaction, the product $5 \mathrm{~h}$ was obtained $(93.7 \mathrm{mg}$, yield $74 \%$ in Hex-EA 75:25). IR spectrum $\left(\mathrm{CHCl}_{3}\right): v_{\max } \mathrm{cm}^{-1}: 2361.5,2336.9,1653.5,1600.1,1506.8,1256.7 ;{ }^{1} \mathrm{H}$ NMR $\left(300 \mathrm{MHz}, \mathrm{CDCl}_{3}, \mathrm{Me}_{4} \mathrm{Si}\right) \delta 3.98(3 \mathrm{H} ; \mathrm{s}), 4.00(3 \mathrm{H} ; \mathrm{s}), 4.77(2 \mathrm{H} ; \mathrm{d}$; $\mathrm{J}=12.6 \mathrm{~Hz}), 5.43(1 \mathrm{H} ; \mathrm{dd} ; \mathrm{J}=12.6$ and $1.0 \mathrm{~Hz}), 5.50(1 \mathrm{H} ; \mathrm{dd} ; \mathrm{J}=12.6 \mathrm{~Hz})$, $6.2(1 \mathrm{H} ; \mathrm{m}), 6.99(1 \mathrm{H} ; \mathrm{d} ; \mathrm{J}=8.1 \mathrm{~Hz}), 7.09(2 \mathrm{H} ; \mathrm{d} ; \mathrm{J}=10.1 \mathrm{~Hz}), 7.26(1 \mathrm{H}$; d; J=2.1 Hz) ,7.30 (1H; dd; J=8.1 and $2.1 \mathrm{~Hz}), 7.51(1 \mathrm{H} ; \mathrm{d} ; \mathrm{J}=15.9 \mathrm{~Hz})$, 7.85 (1H; d; J=15.6 Hz), $8.12(2 \mathrm{H} ; \mathrm{d} ; \mathrm{J}=10,1 \mathrm{~Hz}) ;{ }^{13} \mathrm{C}$ NMR $(75 \mathrm{MHz}$, $\left.\mathrm{CDCl}_{3}, \mathrm{Me}_{4} \mathrm{Si}\right) \delta 55.4 ; 55.9 ; 69.7 ; 110.5 ; 112.9 ; 113.7(\mathrm{x} 2) ; 118.4 ; 119.8$; $122.4 ; 128.2 ; 130.9$ (x2); 131.3; 132.7; 144.0; $149.5 ; 150.2 ; 163.2 ; 188.7$;
ESIMS m/z $325.1[\mathrm{M}+\mathrm{H}]^{+}$. HREIMS $(\mathrm{m} / \mathrm{z}): 324.1358\left[\mathrm{M}^{+}\right]$(calcd for $\left.\mathrm{C}_{20} \mathrm{H}_{20} \mathrm{O}_{4}, 324.1362\right)$.

\section{Statistical analysis}

The one-way ANOVA at 95\% confidence level was used for statistical analysis.

\section{Results and Discussion}

\section{Chemistry (synthesis)}

The synthesis of chalcones $5 \mathrm{a}-\mathrm{h}$ was accomplished by a onepot Claisen-Schmidt condensation [21,22] between the appropriate $O$-allylvanillin 3 and substituted acetophenones $4 \mathrm{a}-\mathrm{h}$, as shown in Scheme 2. O-allylvanillin 3 was prepared via the nucleophilic substitution of vanillin 1 and allylbromide 2 in the presence of potassium carbonate in anhydrous acetone (Scheme 1) [22].

In all the chalcones synthesized, only the trans double bond (on the basis of coupling constant) was obtained. All synthesized compounds were characterized by spectral data (mass, UV, IR and NMR) and were consistent with the structures proposed. The purity of these compounds was ascertained by TLC and spectral analysis.

\section{Biological studies}

These synthesized compounds were evaluated for their in vitro anticancer activity using Sulforhodamine B assays [20]. A preliminary assay against leukemia THP-1 cell line showed that compounds 3 ,

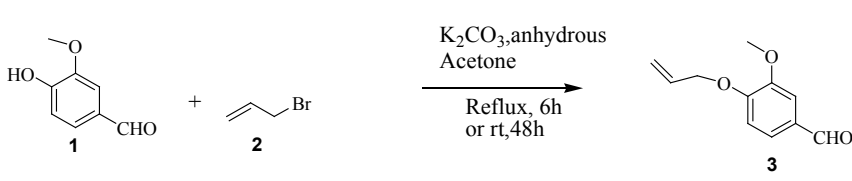

Scheme 1: Synthesis of the intermediate O-allylvanillin 3.

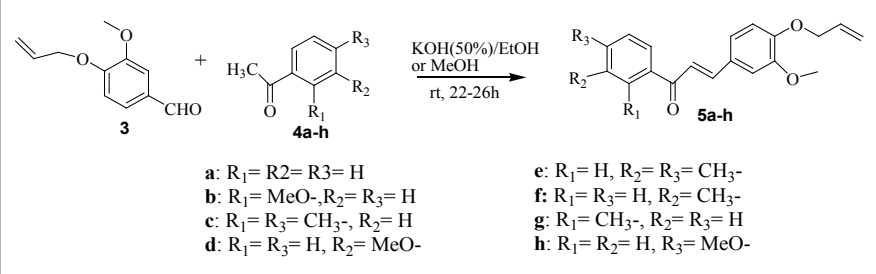

Scheme 2: Synthesis of compounds 5 a-h.

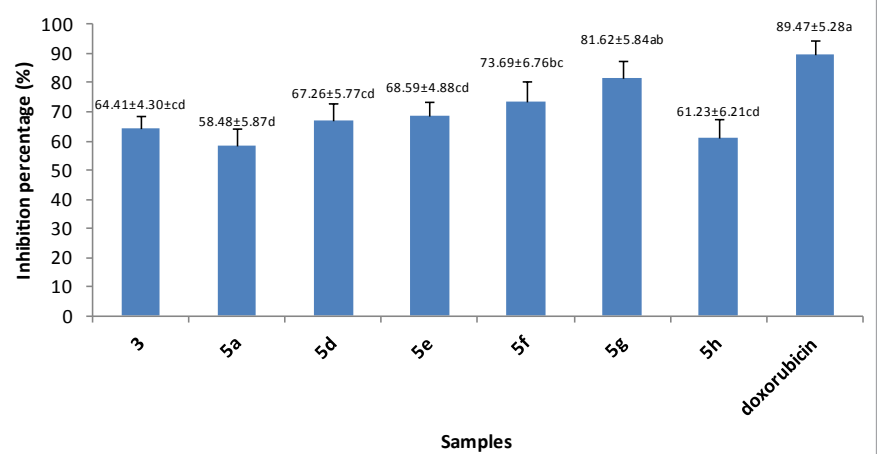

Figure 1: Inhibitory percentage (\%) of compounds at $100 \mu \mathrm{M}$ and doxorubicin $(50 \mu \mathrm{M})$ on leukemia THP-1 cancer cell line. Values mean \pm SD of three experiments; Data with different superscript letters $(a, b, c . .$.$) are significantly$ different $(P<0.05)$. 


\begin{tabular}{|c|c|c|c|c|c|}
\hline \multirow{2}{*}{ Tested samples } & \multicolumn{5}{|c|}{ Cell lines and $\mathrm{IC}_{50}$ values $(\mu \mathrm{M})$} \\
\hline & THP-1 & HL60 & Hep-G2 & DU-145 & MCF-7 \\
\hline 3 & $74.76 \pm 3.27$ & $63.52 \pm 5.2$ & $90.99 \pm 7.72$ & - & $90.11 \pm 7.26$ \\
\hline $5 a$ & $12.80 \pm 1.34$ & $23.52 \pm 2.11$ & - & - & $77.37 \pm 7.12$ \\
\hline $5 d$ & $25.19 \pm 1.94$ & $20.81 \pm 1.97$ & $43.75 \pm 3.42$ & $83.73 \pm 6.43$ & $56.54 \pm 3.78$ \\
\hline $5 e$ & $27.03 \pm 2.03$ & $28.70 \pm 2.37$ & $33.22 \pm 3.07$ & $37.70 \pm 2.71$ & $28.98 \pm 1.91$ \\
\hline $5 f$ & $10.42 \pm 0.68$ & $13.50 \pm 1.14$ & $19.94 \pm 2.15$ & $12.23 \pm 1.19$ & $17.28 \pm 2.02$ \\
\hline $5 \mathrm{~g}$ & $4.76 \pm 0.51$ & $7.90 \pm 0.64$ & $10.12 \pm 0.88$ & $5.21 \pm 0.28$ & $10.32 \pm 0.86$ \\
\hline $5 \mathrm{~h}$ & $27.78 \pm 3.04$ & $37.59 \pm 3.16$ & $53.28 \pm 5.32$ & $36.48 \pm 3.09$ & $45.12 \pm 3.27$ \\
\hline Doxorubicin & $1.44 \pm 0.09$ & $2.17 \pm 0.26$ & $4.31 \pm 0.36$ & $2.59 \pm 0.20$ & $6.00 \pm 0.72$ \\
\hline
\end{tabular}

$(-):>100 \mu \mathrm{M}$

Table 1: Cytotoxicity of the studied compounds towards cancer cell lines.

$5 \mathrm{a}, 5 \mathrm{~d}, 5 \mathrm{e}, 5 \mathrm{f}, 5 \mathrm{~g}$ and $5 \mathrm{~h}($ at $100 \mu \mathrm{M})$ as well as doxorubicin at $50 \mu \mathrm{M}$ were able to inhibit the proliferation of more than $50 \%$ cells (Figure 1). These samples were consequently tested in other cell lines and the results are summarized in Table 1 . It appeared that compounds $5 \mathrm{~d}-\mathrm{h}$ displayed cytotoxic activities with $\mathrm{IC}_{50}$ values below $100 \mu \mathrm{M}$ against the five cancer cell lines. In the US NCI screening program, a compound is generally considered to have in vitro cytotoxic activity, if the $\mathrm{IC}_{50}$ value following incubation between 48 and $72 \mathrm{~h}$ is less than $4 \mu \mathrm{g} / \mathrm{ml}$ or 10 $\mu \mathrm{M}$ [23]. In the present study, $\mathrm{IC}_{50}$ values below or around $10 \mu \mathrm{M}$ were displayed by compounds $5 \mathrm{f}$ against THP- 1 cells $\left(\mathrm{IC}_{50}\right.$ of $\left.10.42 \mu \mathrm{M}\right)$ and $5 \mathrm{~g}$ against THP-1 ( $\mathrm{IC}_{50}$ of $\left.4.76 \mu \mathrm{M}\right), \mathrm{DU}-145\left(\mathrm{IC}_{50}\right.$ of $\left.5.21 \mu \mathrm{M}\right)$, HL60 $\left(\mathrm{IC}_{50}\right.$ of $\left.7.90 \mu \mathrm{M}\right)$, Hep-G2 (IC ${ }_{50}$ of $\left.10.12 \mu \mathrm{M}\right)$ and MCF-7 (IC ${ }_{50}$ of 10.32 $\mu \mathrm{M})$. Also the $\mathrm{IC}_{50}$ values obtained with doxorubicin were below $10 \mu \mathrm{M}$ against the five cancer cell lines tested. The cytotoxicity of compounds $5 \mathrm{~g}$ can be considered good with regards to the US NCI standard. When regarding the structure activity relationship, it appeared that the number and position of methyl group in cycle A of the synthesized chalcones influenced their activities, compound $5 \mathrm{~g}$ with the $-\mathrm{CH}_{3}$ group in position $\mathrm{C}-2$ being more active on almost the five cell lines than compounds $5 \mathrm{a}$ (without any methyl group) and $5 \mathrm{f}$ bearing $-\mathrm{CH}_{3}$ group in $\mathrm{C}-3$ (Table 1). However, $5 \mathrm{e}$ with three $-\mathrm{CH}_{3}$ groups was less active than compound $5 \mathrm{~g}$ and $5 \mathrm{f}$ (only one $-\mathrm{CH}_{3}$ group), but more active than $5 \mathrm{a}$ without a $-\mathrm{CH}_{3}$ group, clearly confirming the influence of the methylation on the activity of the chalcones studied. Also, when comparing the activity of the two most cytotoxic compounds $5 \mathrm{~g}$ and $5 \mathrm{f}$ with those of the methoxylated compounds $5 \mathrm{~d}$ and $5 \mathrm{~h}$, it appeared that a single methylation induced an increase in activity compared to a single methoxylation of the chalcones studied. In addition, it is also clear that, the position of $-\mathrm{CH}_{3}$ and that of $-\mathrm{OCH}_{3}$ groups influence the antiproliferative activities of compounds $5 \mathrm{~d}$ and $5 \mathrm{~h}$. Although the compounds studied did not show very good cytotoxicity, the study provides additional information on structure-activity relationships with chalcones, that could allow future synthesis of more potent derivatives. In future, mechanistic studies such as the effects of compound $5 \mathrm{~g}$ on cell cycle distribution, induction of apoptosis, caspases, and the effects on mitochondrial membrane potential will be carried out to explain the mode of action on this compound.

\section{Conclusion}

In conclusion, we report here a series of new $O$-allylchalcone derivatives prepared by a Claisen-Schmidt condensation reaction [22] and their ability to kill tumor cells in vitro. The mechanisms of cytotoxicity underlying this process remain to be fully elucidated. Previous studies reported in the literature reveal that, flavonoids such as chalcones are known microtubule inhibitors with antimitotic activity [14]. Detailed mechanistic studies and lead optimization of these $O$-allylchalcone derivatives are under investigation. It is intended that results from these studies will assist in elucidating their precise mechanisms of action and provide an approach to develop new potent
$O$-allylchalcone hybrid prototypes for further optimization and development to get new leads for the treatment of cancer.

\section{Acknowledgement}

$\mathrm{BN}, \mathrm{RR}$ and MT are grateful to the Agence Universitaire de la Francophonie (AUF), Natural Sciences and Engineering Research Council of Canada (NSERC) and MATSUMAE for their financial support of this research and for a travel grant to the Department of Chemistry, Université du Québec à Montréal (Canada) and Department of Chemistry, University of Yamagata (Japan), respectively.

\section{References}

1. Di Carlo G, Mascolo N, Izzo AA, Capasso F (1999) Flavonoids: old and new aspects of a class of natural therapeutic drugs. Life Sci 65: 337-353.

2. Batovska D, Parushev S, Stamboliyska B, Tsvetkova I, Ninova M, et al. (2009) Examination of growth inhibitory properties of synthetic chalcones for which antibacterial activity was predicted. Eur J Med Chem 44: 2211-2218.

3. de Barros Machado T, Leal IC, Kuster RM, Amaral AC, Kokis V, et al. (2005) Brazilian phytopharmaceuticals--evaluation against hospital bacteria. Phytother Res 19: 519-525.

4. Larsen M, Kromann H, Kharazmi A, Nielsen SF (2005) Conformationally restricted anti-plasmodial chalcones. Bioorg Med Chem Lett 15: 4858-4861.

5. Frolich S, Schubert C, Bienzle U, Jenett-Siems K (2005) In vitro antiplasmodial activity of prenylated chalcone derivatives of hops (Humulus lupulus) and their interaction with haemin. J Antimicrob Chemother 55: 883-887.

6. Boeck P, Leal PC, Yunes RA, Filho VC, López S, et al. (2005) Antifungal activity and studies on mode of action of novel xanthoxyline-derived chalcones. Arch Pharm (Weinheim) 338: 87-95

7. Cheenpracha S, Karalai C, Ponglimanont C, Subhadhirasakul S, Tewtraku $S$ (2006) Anti-HIV-1 protease activity of compounds from Boesenbergia pandurata. Bioorg Med Chem 14: 1710-1714.

8. Wu JH, Wang $\mathrm{XH}, \mathrm{Yi} \mathrm{YH}$, Lee $\mathrm{KH}$ (2003) Anti-AIDS agents 54. A potent antiHIV chalcone and flavonoids from genus Desmos. Bioorg Med Chem Lett 13 1813-1815.

9. Herencia F, Ferrandiz ML, Ubeda A, Guillen I, Dominguez JN, et al. (2001) 4-dimethylamino-3',4'-dimethoxychalcone downregulates iNOS expression and exerts anti-inflammatory effects. Free Radic Biol Med 30: 43-50.

10. Rojas J, Paya M, DomÃnguez JN, Ferrandiz ML (2003) ttCH, a selective inhibitor of inducible nitric oxide synthase expression with antiarthritic properties. Eur $J$ Pharmacol 465: 183-189.

11. Ngameni B, Touaibia M, Belkaid A, Ambassa P, Watchueng J, et al. (2007) Inhibition of matrix metalloproteinase-2 secretion by chalcones from the twigs of Dorstenia barteri Bureau. ARKIVOC ix: 91-103.

12. Go ML, Wu X, Liu XL (2005) Chalcones: an update on cytotoxic and chemoprotective properties. Curr Med Chem 12: 481-499.

13. Vanhoecke BW, Delporte F, Van Braeckel E, Heyerick A, Depypere HT, et al. (2005) A safety study of oral tangeretin and xanthohumol administration to laboratory mice. In vivo 19: 103-107.

14. Lawrence NJ, McGown AT (2005) The chemistry and biology of antimitotic chalcones and related enone systems. Curr Pharm Des 11: 1679-1693.

15. Calliste CA, Le Bail JC, Trouillas P, Pouget C, Habrioux G, et al. (2001) Chalcones: structural requirements for antioxidant, estrogenic and antiproliferative activities. Anticancer Res 21: 3949-3956. 
Citation: Ngameni B, Kuete V, Ambassa P, Justin K, Marlyse ML, et al. (2013) Synthesis and Evaluation of Anticancer Activity of O-allylchalcone Derivatives. Med chem 3: 233-237. doi:10.4172/2161-0444.1000144

16. Park EJ, Park HR, Lee JS, Kim J (1998) Licochalcone A: an inducer of cell differentiation and cytotoxic agent from Pogostemon cablin. Planta Med 64: 464-466.

17. Adibi H, Mojarrad JS, Asgharloo H, Zarrini G (2011) Synthesis, in vitro antimicrobial and antioxidant activities of chalcone and flavones derivatives holding allylic substitutions, Med Chem Res 20: 1318-1324.

18. Doan TN, Tran DT (2011) Synthesis, antioxidant and antimicrobial activities of a novel series of chalcones and allylic chalcones, Pharmacology \&Pharmacy 2: $282-288$.

19. Mizuno CS, Paul S, Suh N, Rimando AM (2010) Synthesis and biological evaluation of retinoid-chalcones as inhibitors of colon cancer cell growth. Bioorg Med Chem Lett 20: 7385-7387.
20. Skehan P, Storeng R, Scudiero D, Monks A, McMahon J, et al. (1990) New colorimetric cytotoxicity assay for anticancer-drug screening. J Natl Cancer Inst 82: $1107-1112$.

21. Modzelewska A, Pettit C, Achanta G, Davidson NE, Huang P, et al. (2006) Anticancer activities of novel chalcone and bis-chalcone derivatives. Bioorg Med Chem 14: 3491-3495.

22. Mukherjee S, Kumar V, Prasad AK, Raj HG, Bracke ME, et al. (2001) Synthetic and biological activity evaluation studies on novel 1,3-diarylpropenones. Bioorg Med Chem 9: 337-345

23. Kuete V, Ngameni B, Wiench B, Krusche B, Horwedel C, et al. (2011) Cytotoxicity and mode of action of four naturally occuring flavonoids from the genus Dorstenia: gancaonin Q, 4-hydroxylonchocarpin, 6-prenylapigenin, and 6,8-diprenyleriodictyol. Planta Med 77: 1984-1989. 